\title{
Existence and multiplicity of positive bound states for Schrödinger equations
}

\author{
Sun Sheng, Fanglei Wang* and Tianqing An
}

${ }^{\text {*Correspondence: }}$

wang-fanglei@hotmail.com

College of Science, Hohai University,

Nanjing, 210098, P.R. China

\begin{abstract}
In this paper, we study the existence and multiplicity of positive bound states of non-autonomous systems of nonlinear Schrödinger equations. The proof is based on the fixed point theorems in a cone.
\end{abstract}

MSC: 34B15; 35J20

Keywords: nonlinear Schrödinger systems; positive solutions; fixed point theorems

\section{Introduction}

Because of the important background in nonlinear optics and other fields, many authors pay more attention to the study of different types of vector nonlinear Schrödinger equations, we refer the readers to [1-5]. Most of these results have been proven using critical point theory, variational approaches or a fixed point theorem. More recently, Chu has applied another topological approach, a nonlinear alternative principle of Leray-Schauder, to establish some new existence results for the following Schrödinger equations

$$
-\ddot{u}(x)+a(x) u(x)=b(x) f(u(x))+e(x),
$$

where $a \in L^{\infty}\left(R, R^{N}\right)$ is nonnegative almost everywhere, $e \in L^{1}\left(R, R^{N}\right)$. The author considered two different cases. One is the singular case, that is, $f \in C\left(R^{N} \backslash\{0\}, R^{N}\right)$ and

$$
\lim _{u \rightarrow 0} f_{i}(u)=+\infty, \quad i=1,2, \ldots, n .
$$

The other is the regular case, that is, $f \in C\left(R^{N}, R^{N}\right)$. However, the references [4-6] are not concerned with multiplicity of positive solutions for the scalar Schrödinger equation or system.

Motivated by the study of solitary wave solutions, in this paper we mainly aim to study the existence and multiplicity of positive bound states of the more general system of nonautonomous Schrödinger equations

$$
\left\{\begin{array}{l}
-\ddot{u}(x)+A(x) u(x)=C(x) F(u(x)), \\
\lim _{|x| \rightarrow \infty} u(x)=\lim _{|x| \rightarrow \infty} \dot{u}(x)=0, \\
\int_{-\infty}^{+\infty} u^{2}(x) d x+\int_{-\infty}^{+\infty} \dot{u}^{2}(x) d x<+\infty,
\end{array}\right.
$$

2013 Sheng et al.: licensee Springer. This is an Open Access article distributed under the terms of the Creative Commons Attribution License (http://creativecommons.org/licenses/by/2.0), which permits unrestricted use, distribution, and reproduction in any medium, provided the original work is properly cited. 
where

$$
\begin{aligned}
& u(x)=\left(\begin{array}{c}
u_{1}(x) \\
u_{2}(x) \\
\vdots \\
u_{n}(x)
\end{array}\right), \quad A(x)=\left(\begin{array}{cccc}
a_{11}(x) & a_{12}(x) & \cdots & a_{1 n}(x) \\
a_{21}(x) & a_{22}(x) & \cdots & a_{2 n}(x) \\
\vdots & \vdots & \ddots & \vdots \\
a_{n 1}(x) & a_{n 2}(x) & \cdots & a_{n n}(x)
\end{array}\right), \\
& C(x)=\left(\begin{array}{cccc}
c_{1}(x) & 0 & \cdots & 0 \\
0 & c_{2}(x) & \cdots & 0 \\
\vdots & \vdots & \ddots & \vdots \\
0 & 0 & \cdots & c_{n}(x)
\end{array}\right), \quad F(u(x))=\left(\begin{array}{c}
f_{1}\left(u_{1}(x), u_{2}(x), \ldots, u_{n}(x)\right) \\
f_{2}\left(u_{1}(x), u_{2}(x), \ldots, u_{n}(x)\right) \\
\vdots \\
f_{n}\left(u_{1}(x), u_{2}(x), \ldots, u_{n}(x)\right)
\end{array}\right) .
\end{aligned}
$$

The methods used here are the Krasnoselskii fixed point theorem and the LeggettWilliams fixed point theorem together with a compactness criterion due to Zima.

We organize the paper as follows. In Section 2, we give some preliminaries; in Section 3, we discuss the existence and multiplicity of positive solutions for (1).

\section{Preliminaries}

For convenience, we assume that the following conditions hold throughout this paper.

(H1) $a_{i j} \in L^{\infty}(R, R)$, and $a_{i j}(x)$ satisfies the following property

$$
a_{i j}(x)=\left\{\begin{array}{l}
a_{i i}(x) \geq 0, \quad \text { and } \inf a_{i i}(x)>0 \text { if } i=j, \\
a_{i j}(x) \leq 0, \quad \text { and } \operatorname{Supp}\left(a_{i j}\right) \text { is a nonempty compact set if } i \neq j .
\end{array}\right.
$$

(H2) The support of $c_{i}(x)>0$ denoted by $\operatorname{Supp}\left(c_{i}\right)$ is a nonempty compact set, and

$$
0<\int_{M} G_{i}(s, s) c_{i}(s) d s<+\infty
$$

(H3) $f_{i}(u(x)) \in C\left(R^{N}, R^{+}\right)$is continuous, and the following notations are introduced:

$$
f_{i, 0}=\lim _{u \rightarrow 0} \frac{f_{i}(u)}{\sum_{i=1}^{n} u_{i}}, \quad f_{i, \infty}=\lim _{u \rightarrow \infty} \frac{f_{i}(u)}{\sum_{i=1}^{n} u_{i}} .
$$

Since (H1) holds, then for the homogeneous problem

$$
\left\{\begin{array}{l}
-\ddot{\phi}(x)+a_{i i}(x) \phi(x)=0 \\
\phi(-\infty)=0, \quad \phi(+\infty)=0
\end{array}\right.
$$

the associated Green's function is expressed by

$$
G_{i}(x, s)= \begin{cases}\phi_{i}^{1}(x) \phi_{i}^{2}(s), & -\infty<x \leq s<+\infty \\ \phi_{i}^{1}(s) \phi_{i}^{2}(x), & -\infty<s \leq x<+\infty\end{cases}
$$

where $\phi_{i}^{1}, \phi_{i}^{2}$ are solutions such $\phi_{i}^{1}(-\infty)=0, \phi_{i}^{2}(+\infty)=0$. Moreover, $\phi_{i}^{1}, \phi_{i}^{2}$ can be chosen as positive increasing and positive decreasing functions, respectively. Note that $\phi_{i}^{1}, \phi_{i}^{2}$ 
intersect at a unique point $x_{0}$. Therefore, we can define a function $p_{i}(x) \in B C(R)$ by

$$
p_{i}(x)= \begin{cases}\frac{1}{\phi_{i}^{2}(x)}, & x \leq x_{0}, \\ \frac{1}{\phi_{i}^{1}(x)}, & x>x_{0},\end{cases}
$$

where $B C(R)$ denotes the space of bounded continuous functions.

Lemma 2.1 [4] For each $i=1,2, \ldots, n$, Green's function $G_{i}(x, s)$ satisfies the following properties:

(i) $G_{i}(x, s)>0$ for every $(x, s) \in R \times R$;

(ii) $G_{i}(x, s) \leq G_{i}(s, s)$ for every $(x, s) \in R \times R$;

(iii) Given a nonempty compact subset $P \subset R$, we have

$$
\begin{aligned}
G_{i}(x, s) & \geq m_{i}(P) p_{i}(s) G_{i}(s, s) \quad \text { for all }(x, s) \in P \times R, \\
\text { where } m_{i}(P) & =\min \left\{\phi_{i}^{1}(\inf P), \phi_{i}^{2}(\inf P)\right\} .
\end{aligned}
$$

Lemma 2.2 [4] Assume that (H1) holds and $e(x) \in L^{1}(R)$. Then the unique solution of

$$
\left\{\begin{array}{l}
-\ddot{u}_{i}(x)+a_{i i}(x) u_{i}(x)=e(x), \\
u_{i}(-\infty)=0, \quad u_{i}(+\infty)=0
\end{array}\right.
$$

belongs to $H^{1}(R)$, and the solution can be expressed as

$$
u_{i}(x)=\int_{R} G_{i}(x, s) e(s) d s .
$$

The proof of our main results is based on the following fixed points, which can be found in [7].

Lemma 2.3 Let $E$ be a Banach space, and let $K \subset E$ be a cone in $E$. Assume that $\Omega_{1}, \Omega_{2}$ are open subsets of $E$ with $0 \in \Omega_{1}, \bar{\Omega}_{1} \subset \Omega_{2}$, and let $T: K \cap\left(\bar{\Omega}_{2} \backslash \Omega_{1}\right) \rightarrow K$ be a completely continuous operator such that either

(i) $\|T u\| \leq\|u\|, u \in K \cap \partial \Omega_{1}$ and $\|T u\| \geq\|u\|, u \in K \cap \partial \Omega_{2}$; or

(ii) $\|T u\| \geq\|u\|, u \in K \cap \partial \Omega_{1}$ and $\|T u\| \leq\|u\|, u \in K \cap \partial \Omega_{2}$.

Then $T$ has a fixed point in $K \cap\left(\bar{\Omega}_{2} \backslash \Omega_{1}\right)$.

Let $E$ be a real Banach space and $P$ be a cone in $E$. A map $\alpha$ is said to be a nonnegative continuous concave functional on $P$ if

$$
\alpha: P \rightarrow[0,+\infty)
$$

is continuous and

$$
\alpha(t x+(1-t) y) \geq t \alpha(x)+(1-t) \alpha(y)
$$

for all $x, y \in P$ and $t \in[0,1]$. 
For numbers $a, b$ such that $0<a<b$, letting $\alpha$ be a nonnegative continuous concave functional on $P$, we define the following convex sets:

$$
P_{a}=\{x \in P:\|x\|<a\}
$$

and

$$
P(\alpha, a, b)=\{x \in P: a \leq \alpha(x),\|x\| \leq b\} .
$$

Lemma 2.4 Let $T: \bar{P}_{c} \rightarrow \bar{P}_{c}$ be completely continuous and $\alpha$ be a nonnegative continuous concave functional on $P$ such that $\alpha(x) \leq\|x\|$ for all $x \in \bar{P}_{c}$. Suppose that there exist $0<d<$ $a<b \leq c$ such that

(i) $\{x \in P(\alpha, a, b): \alpha(x)>a\} \neq \emptyset$ and $\alpha(T x)>a$ for $x \in P(\alpha, a, b)$;

(ii) $\|T x\|<d$ for $\|x\| \leq d$;

(iii) $\alpha(T x)>a$ for $x \in P(\alpha, a, c)$ with $\|T x\|>b$.

Then $T$ has at least three fixed points $x_{1}, x_{2}, x_{3}$ satisfying

$$
\begin{aligned}
& \left\|x_{1}\right\|<d, \quad a<\alpha\left(x_{2}\right), \\
& \left\|x_{3}\right\|>d \quad \text { and } \quad \alpha\left(x_{3}\right)<a .
\end{aligned}
$$

In addition, the following compactness criterion proved by Zima in [8] is also used in our proof.

Lemma 2.5 Let $\Omega \subset B C(R)$. Let us assume that the functions $u \in \Omega$ are equicontinuous in each compact interval of $R$ and that for all $u \in \Omega$, we have

$$
|u(x)| \leq \xi(x), \quad \forall x \in R
$$

where $\xi \in B C(R)$ verifies

$$
\lim _{|x| \rightarrow+\infty} \xi(x)=0
$$

Then $\Omega$ is relatively compact.

\section{Main results}

From now on, we assume that $M=\bigcup_{i \neq j} \operatorname{Supp}\left(a_{i j}\right) \cup \operatorname{Supp}\left(c_{i}\right)$ is a nonempty compact set. Let $E$ denote the Banach space $\overbrace{B C(R) \times B C(R) \times \cdots \times B C(R)}^{n}$ with the norm $\|u\|=\sum_{i=1}^{n}\left|u_{i}\right|_{\infty}$, $\left|u_{i}\right|_{\infty}=\max _{x \in M}\left|u_{i}(x)\right|$ for $u=\left(u_{1}, u_{2}, \ldots, u_{n}\right) \in E$. Define a cone $K \subset E$ as

$$
K=\left\{u=\left(u_{1}, u_{2}, \ldots, u_{n}\right) \in E: u_{i}(x) \geq 0 \text { and } \min _{x \in M} \sum_{i=1}^{n} u_{i}(x) \geq \delta\|u\|\right\},
$$

where $\delta=\min _{i=1,2, \ldots, n}\left\{m_{i} p_{0}^{i}\right\} \in(0,1), p_{0}^{i}=\inf _{M} p_{i}(x)$ and the constants $m_{i} \equiv m_{i}(M), i=$ $1,2, \ldots, n$, are defined by property (iii) of Lemma 2.1. Since $M$ is compact, then $p_{0}^{i}>0$, $i=1,2, \ldots, n$. Moreover, from (iii) of Lemma 2.1 it follows that $m_{i} p_{0}^{i}<1$ for $i=1,2, \ldots, n$. 
Let $T: K \rightarrow E$ be a map with components $\left(T_{1}, \ldots, T_{n}\right)$ defined by

$$
T_{i}(u)(x)=\int_{M} G_{i}(x, s)\left[-\sum_{j \neq i} a_{i j} u_{j}+c_{i}(s) f_{i}(u(s))\right] d s .
$$

A fixed point of $T$ is a solution of (1) which belongs to $\overbrace{H^{1}(R) \times \cdots \times H^{1}(R)}^{n}$.

Lemma 3.1 Assume that $(\mathrm{H} 1)-(\mathrm{H} 3)$ hold. Then $T(K) \subset K$, and $T: K \rightarrow K$ is completely continuous.

Proof The continuity is trivial. Since $M$ is compact, there exists a point $x_{m}$ where $\min _{x \in M} T_{i}(u)(x)$ is attained. Then, for any $x \in R$, we have

$$
\begin{aligned}
T_{i}(u)\left(x_{m}\right) & =\int_{M} G_{i}(x, s)\left[-\sum_{j \neq i} a_{i j} u_{j}+c_{i}(s) f_{i}(u(s))\right] d s \\
& \geq m_{i} \int_{M} p_{i}(s) G_{i}(s, s)\left[-\sum_{j \neq i} a_{i j} u_{j}+c_{i}(s) f_{i}(u(s))\right] d s \\
& \geq m_{i} p_{0}^{i} \int_{M} G_{i}(s, s)\left[-\sum_{j \neq i} a_{i j} u_{j}+c_{i}(s) f_{i}(u(s))\right] d s \\
& \geq m_{i} p_{0}^{i} \int_{M} G_{i}(x, s)\left[-\sum_{j \neq i} a_{i j} u_{j}+c_{i}(s) f_{i}(u(s))\right] d s \\
& \geq m_{i} p_{0}^{i} T_{i}(u)(x),
\end{aligned}
$$

namely,

$$
\min _{x \in M} T_{i}(u)(x) \geq m_{i} p_{0}^{i} T_{i}(u)(x) \geq \delta T_{i}(u)(x) .
$$

Therefore, it is clear that $T(K) \subset K$.

Finally, we prove that each component of $T$ is compact. Let $\Omega \subset K$ be a bounded set, then there exists a constant $C>0$ which is uniformly bounded for its element. Since the derivative is bounded in compacts, the functions of $T_{i}(\Omega)$ are equicontinuous on each compact interval. On the other hand, for any $u \in \Omega$,

$$
\left|T_{i}(u)(x)\right| \leq C \int_{R} G_{i}(x, s)\left(-\sum_{j \neq i} a_{i j}(s)\right) d s+\max _{\|u\| \leq C} f_{i}(u) \int_{R} G_{i}(x, s) c_{i}(s) d s=\xi(x) .
$$

Theorem 3.2 Assume that (H1)-(H3) hold. In addition, $a_{i j}(x)(i \neq j)$ satisfies

$$
\int_{M} G_{i}(s, s)\left(-\sum_{j \neq i} a_{i j}\right) d s<\frac{1}{2 n} .
$$

(a) If $f_{i, 0}=0, f_{i_{0}, \infty}=\infty$ for some $i_{0} \in\{1,2, \ldots, n\}$, then (1) has at least one positive solution.

(b) If $f_{i, \infty}=0, f_{i_{0}, 0}=\infty$ for some $i_{0} \in\{1,2, \ldots, n\}$, then (1) has at least one positive solution. 
Proof (a) On the one hand, since $f_{i, 0}=0$, then there exists $r>0$ such that

$$
f_{i}(u) \leq \epsilon \sum_{i=1}^{n} u_{i} \quad \text { for } 0<u_{1}+\cdots+u_{n} \leq r
$$

where $\epsilon>0$ is sufficiently small such that

$$
\epsilon \int_{M} G_{i}(s, s) c_{i}(s) d s<\frac{1}{2 n} .
$$

Set $\Omega_{r}=\{u \in E:\|u\|<r\}$. Then, for any $u \in \partial \Omega_{r} \cap K$, we have

$$
\begin{aligned}
T_{i}(u)(x) & =\int_{M} G_{i}(x, s)\left[-\sum_{j \neq i} a_{i j} u_{j}+c_{i}(s) f_{i}(u(s))\right] d s \\
& \leq \int_{M} G_{i}(s, s)\left[-\sum_{j \neq i} a_{i j}(s) u_{j}(s)+c_{i}(s) \epsilon \sum_{i=1}^{n} u_{i}(s)\right] d s \\
& \leq\left[\int_{M} G_{i}(s, s)\left(-\sum_{j \neq i} a_{i j}(s)\right) d s+\epsilon \int_{M} G_{i}(s, s) c_{i}(s) d s\right]\|u\| \\
& \leq \frac{1}{n}\|u\| .
\end{aligned}
$$

Furthermore, for any $u \in \partial \Omega_{r} \cap K$, we have

$$
\|T(u)\|=\sum_{i=1}^{n}\left|T_{i}(u)\right|_{\infty} \leq\|u\| .
$$

On the other hand, since $f_{i_{0}, \infty}=\infty$ for some $i_{0} \in\{1,2, \ldots, n\}$, then there exists $\bar{R}$ such that

$$
f_{i_{0}}(u) \geq \eta \sum_{i=1}^{n} u_{i} \quad \text { for } \sum_{i=1}^{n} u_{i} \geq \bar{R}
$$

where $\eta>0$ is sufficiently large such that

$$
\eta \delta^{2} \int_{M} G_{i_{0}}(s, s) c_{i_{0}}(s) d s>1
$$

Let $R=\max \left\{2 r, \frac{\bar{R}}{\delta}\right\}$ and set $\Omega_{R}=\{u \in E:\|u\|<R\}$. Then, for any $u \in K \cap \partial \Omega_{2}$, $\min _{x \in M} \sum_{i=1}^{n} u_{i}(x) \geq \delta\|u\| \geq \bar{R}$, and we have

$$
\begin{aligned}
T_{i_{0}}(u)(x) & =\int_{M} G_{i_{0}}(x, s)\left[-\sum_{j \neq i_{0}} a_{i_{0}} u_{j}+c_{i_{0}}(s) f_{i_{0}}(u(s))\right] d s \\
& \geq m_{i_{0}} p_{0}^{i_{0}} \int_{M} G_{i_{0}}(s, s) c_{i_{0}}(s) \eta \sum_{i=1}^{n} u_{i}(s) d s \\
& \geq \eta \delta^{2} \int_{M} G_{i_{0}}(s, s) c_{i_{0}}(s) d s\|u\| \\
& >\|u\| .
\end{aligned}
$$


Furthermore, we have

$$
\|T(u)\|>\|u\| \quad \text { for } u \in K \cap \partial \Omega_{R} .
$$

Now by Lemma $2.3, T$ has a fixed point $u=\left(u_{1}, u_{2}, \ldots, u_{n}\right) \in K \cap\left(\bar{\Omega}_{R} \backslash \Omega_{r}\right)$, namely, (1) has a positive solution.

(b) On the one hand, since $f_{i_{0}, 0}=\infty$ for some $i_{0} \in\{1,2, \ldots, n\}$, there exists $r>0$ such that

$$
f_{i_{0}}(u) \geq \eta \sum_{i=1}^{n} u_{i} \quad \text { for } 0 \leq \sum_{i=0}^{n} u_{i} \leq r
$$

where $\eta>0$ is sufficiently large such that

$$
\eta \delta^{2} \int_{M} G_{i_{0}}(s, s) c_{i_{0}}(s) d s>1
$$

Set $\Omega_{r}=\{u \in E:\|u\|<r\}$. Then, for any $u \in K \cap \partial \Omega_{r}$, we have

$$
\begin{aligned}
T_{i_{0}}(u)(x) & =\int_{M} G_{i_{0}}(x, s)\left[-\sum_{j \neq i_{0}} a_{i_{0} j} u_{j}+c_{i_{0}}(s) f_{i_{0}}(u(s))\right] d s \\
& \geq m_{i_{0}} p_{0}^{i_{0}} \int_{M} G_{i_{0}}(s, s) c_{i_{0}}(s) \eta \sum_{i=1}^{n} u_{i}(s) d s \\
& \geq \eta \delta^{2} \int_{M} G_{i_{0}}(s, s) c_{i_{0}}(s) d s\|u\| \\
& >\|u\| .
\end{aligned}
$$

Furthermore, we have

$$
\|T(u)\|>\|u\| \quad \text { for } u \in K \cap \partial \Omega_{r} .
$$

On the other hand, since $f_{i, \infty}=0$, then there exists $\bar{R}$ such that

$$
f_{i}(u) \leq \epsilon \sum_{i=1}^{n} u_{i} \quad \text { for } u_{1}+\cdots+u_{n} \geq \bar{R}
$$

where $\epsilon>0$ is sufficiently small such that

$$
\epsilon \int_{M} G_{i}(s, s) c_{i}(s) d s<\frac{1}{2 n} .
$$

Let $R=\max \left\{2 r, \frac{\bar{R}}{\delta}\right\}$ and set $\Omega_{R}=\{u \in E:\|u\|<R\}$. Then, for any $u \in K \cap \partial \Omega_{R}$, $\min _{x \in M} \sum_{i=1}^{n} u_{i}(x) \geq \delta\|u\| \geq \bar{R}$, and we have

$$
\begin{aligned}
T_{i}(u)(x) & =\int_{M} G_{i}(x, s)\left[-\sum_{j \neq i} a_{i j} u_{j}+c_{i}(s) f_{i}(u(s))\right] d s \\
& \leq \int_{M} G_{i}(s, s)\left[-\sum_{j \neq i} a_{i j}(s) u_{j}(s)+c_{i}(s) \epsilon \sum_{i=1}^{n} u_{i}(s)\right] d s
\end{aligned}
$$




$$
\begin{aligned}
& \leq\left[\int_{M} G_{i}(s, s)\left(-\sum_{j \neq i} a_{i j}(s)\right) d s+\epsilon \int_{M} G_{i}(s, s) c_{i}(s) d s\right]\|u\| \\
& \leq \frac{1}{n}\|u\| .
\end{aligned}
$$

Furthermore, for any $u \in \partial \Omega_{R} \cap K$, we have

$$
\|T(u)\|=\sum_{i=1}^{n}\left|T_{i}(u)\right|_{\infty} \leq\|u\| .
$$

Now, by Lemma 2.3, $T$ has a fixed point $u=\left(u_{1}, u_{2}, \ldots, u_{n}\right) \in K \cap\left(\bar{\Omega}_{R} \backslash \Omega_{r}\right)$, namely, (1) has a positive solution.

Corollary 3.3 Assume that (H1)-(H3) hold. $a_{i j}(x)(i \neq j)$ satisfies

$$
\int_{M} G_{i}(s, s)\left(-\sum_{j \neq i} a_{i j}\right) d s<\frac{1}{2 n}
$$

In addition, the following conditions hold.

(H4) If there exist constants $\widehat{R}, \rho>0$ such that for some $i_{0} \in\{1,2, \ldots, n\}$,

$$
f_{i_{0}}(u) \geq \rho \widehat{R} \quad \text { for } \delta \widehat{R} \leq \sum_{i=1}^{n} u_{i} \leq \widehat{R},
$$

where $\rho$ satisfies

$$
\rho \delta \int_{M} G_{i_{0}}(s, s) c_{i_{0}}(s) d s>1
$$

(H5) $f_{i, 0}=0, f_{i, \infty}=0$.

Then (1) has at least two positive solutions.

Corollary 3.4 Assume that (H1)-(H3) hold. $a_{i j}(x)(i \neq j)$ satisfies

$$
\int_{M} G_{i}(s, s)\left(-\sum_{j \neq i} a_{i j}\right) d s<\frac{1}{2 n}
$$

In addition, the following conditions hold.

(H6) If there exist constants $\widetilde{R}, \vartheta>0$ such that

$$
f_{i}(u) \leq \vartheta \widetilde{R} \quad \text { for } \delta \widetilde{R} \leq \sum_{i=1}^{n} u_{i} \leq \widetilde{R},
$$

where $\vartheta$ satisfies

$$
\vartheta \int_{M} G_{i}(s, s) c_{i}(s) d s<\frac{1}{2 n}
$$

(H7) $f_{i_{0}, 0}=\infty, f_{j_{0}, \infty}=\infty$ for some $i_{0}, j_{0} \in\{1,2, \ldots, n\}$.

Then (1) has at least two positive solutions. 
Theorem 3.5 Assume that $(\mathrm{H} 1)-(\mathrm{H} 3)$ hold and $a_{i j}(x)(i \neq j)$ satisfies

$$
\int_{M} G_{i}(s, s)\left(-\sum_{j \neq i} a_{i j}\right) d s<\frac{1}{2 n}
$$

In addition, there exist numbers $a, c$ and $d$ with $0<d<a<\frac{c}{4}$ such that the following conditions are satisfied:

(H8) $f_{i}(u)<\frac{1}{2 n \int_{M} G_{i}(s, s) c_{i}(s) d s} d$ for $u_{i} \geq 0$ and $0 \leq \sum_{i=1}^{n} u_{i}<d$;

(H9) there exists $i_{0} \in\{1,2, \ldots, n\}$ such that

$$
f_{i_{0}}(u(x))>\frac{a}{\delta^{2} \Gamma} \quad \text { for } x \in M, u_{i} \geq 0 \text { and } \sum_{i=1}^{n} u_{i} \in\left[a, \frac{a}{\delta}\right]
$$

where $\Gamma=\min \left\{\int_{M} G_{i}(s, s) c_{i}(s) d s\right\}$

(H10) $f_{i}(u) \leq \frac{1}{2 n \int_{M} G_{i}(s, s) c_{i}(s) d s} c$ for $u_{i} \geq 0$ and $\sum_{i=1}^{n} u_{i}<c$.

Then (1) has at least three positive solutions.

Proof For $u=\left(u_{1}, u_{2}, \ldots, u_{n}\right) \in K$, define

$$
\alpha(u)=\min _{x \in M}\left(u_{1}(x)+u_{2}(x)+\cdots+u_{n}(x)\right),
$$

then it is easy to know that $\alpha$ is a nonnegative continuous concave functional on $K$ with $\alpha(u) \leq\|u\|$ for $u \in K$.

Set $b=\frac{a}{\delta}$. First, we show that $T: \bar{K}_{c} \rightarrow \bar{K}_{c}$ with $c>b$. For any $u \in \bar{K}_{c}$, we have

$$
\begin{aligned}
T_{i}(u)(x) & =\int_{R} G_{i}(x, s)\left[-\sum_{j \neq i} a_{i j} u_{j}+c_{i}(s) f_{i}(u(s))\right] d s \\
& =\int_{M} G_{i}(x, s)\left[-\sum_{j \neq i} a_{i j} u_{j}+c_{i}(s) f_{i}(u(s))\right] d s \\
& \leq \int_{M} G_{i}(s, s)\left[-\sum_{j \neq i} a_{i j} u_{j}+c_{i}(s) f_{i}(u(s))\right] d s \\
& \leq \int_{M} G_{i}(s, s)\left[-\sum_{j \neq i} a_{i j} u_{j}+c_{i}(s) \frac{c}{2 n \int_{M} G_{i}(s, s) c_{i}(s) d s}\right] d s \\
& \leq \frac{c}{2 n}+\frac{c}{2 n} \\
& =\frac{c}{n} .
\end{aligned}
$$

So $\|T(u)\|=\sum_{i=1}^{n}\left|T_{i}(u)\right|_{\infty} \leq c$.

In a similar way, we also can prove that $T: \bar{K}_{d} \rightarrow K_{d}$. Then (ii) of Lemma 2.4 holds.

Next, we shall show that (i) of Lemma 2.4 is satisfied. It is clearly seen that $u=$ $\left(\frac{a+b}{2 n}, \ldots, \frac{a+b}{2 n}\right) \in\left\{u=\left(u_{1}, u_{2}, \ldots, u_{n}\right) \in K(\alpha, a, b): \alpha(u)>a\right\}$. Then, for any $u \in K(\alpha, a, b)$ and $x \in M$, it is easy to obtain that

$$
b \geq \sum_{i=1}^{n}\left|u_{i}\right|_{\infty} \geq \sum_{i=1}^{n} u_{i}(x) \geq \min _{x \in M}\left(\sum_{i=1}^{n} u_{i}(x)\right)=\alpha(u)>a .
$$


Then, by (H9), we can have

$$
\begin{aligned}
\alpha(T(u)(x)) & =\min _{x \in M}\left(\sum_{i=1}^{n} T_{i}(u)(x)\right) \\
& \geq \min _{x \in M} T_{i_{0}}(u)(x) \\
& \geq \delta \max _{x \in M} T_{i_{0}}(u)(x) \\
& \geq \delta \int_{M} G_{i_{0}}(x, s)\left[-\sum_{j \neq i_{0}} a_{i_{0} j} u_{j}+c_{i_{0}}(s) f_{i_{0}}(u(s))\right] d s \\
& \geq \delta \int_{M} m_{i_{0}} p_{i_{0}}(s) G_{i_{0}}(s, s) c_{i_{0}}(s) f_{i_{0}}(u(s)) d s \\
& \geq \delta^{2} \int_{M} G_{i_{0}}(s, s) c_{i_{0}}(s) \frac{a}{\delta^{2} \Gamma} d s \\
& =a .
\end{aligned}
$$

Finally, we verify that (iii) of Lemma 2.4 is satisfied. Suppose that $u \in K(\alpha, a, c)$ with $\|T(u)\|>b$, then we can have

$$
\begin{aligned}
\alpha(T(u)) & =\min _{M}\left(\sum_{i=1}^{n} T_{i}(u)(x)\right) \\
& \geq \delta\|T(u)\| \\
& >b \delta=a .
\end{aligned}
$$

From the above, the hypotheses of Leggett-Williams theorem are satisfied. Hence (1) has at least three positive solutions $u^{1}, u^{2}$ and $u^{3}$ such that $\left\|u^{1}\right\|<d, a<\min _{x \in M}\left(\sum_{i=1}^{n} u_{i}^{2}(x)\right)$, and $\left\|u^{3}\right\|>d$ with

$$
\min _{x \in M}\left(\sum_{i=1}^{n} u_{i}^{3}(x)\right)<a .
$$

\section{Competing interests}

The authors declare that they have no competing interests.

\section{Authors' contributions}

SS drafted the manuscript. FW and TA gave some suggestions to improve the manuscript. All authors typed, read and approved the final manuscript.

\section{Received: 5 September 2013 Accepted: 19 November 2013 Published: 12 Dec 2013}

\section{References}

1. Ambrosetti, A, Colorado, E: Standing waves of some coupled nonlinear Schrödinger equations. J. Lond. Math. Soc. 75, 67-82 (2007)

2. Ambrosetti, A: Remarks on some systems of nonlinear Schrödinger equations. J. Fixed Point Theory Appl. 4, 35-46 (2008)

3. Ambrosetti, A, Cerami, G, Ruiz, D: Solitons of linearly coupled systems of semilinear non-autonomous equations on $R^{n}$. J. Funct. Anal. 254, 2816-2845 (2008)

4. Torres, PJ: Guided waves in a multi-layered optical structure. Nonlinearity 19, 2103-2113 (2006)

5. Belmonte-Beitia, J, Pérez-García, VM, Torres, PJ: Solitary waves for linearly coupled nonlinear Schrödinger equations with inhomogeneous coefficients. J. Nonlinear Sci. 19, 437-451 (2009)

6. Chu, J: Positive bound states of systems of nonlinear Schrödinger equations. Nonlinear Anal. 72, 1983-1992 (2010)

7. Guo, D, Lakshmikantham, V: Nonlinear Problems in Abstract Cones. Academic Press, New York (1988)

8. Zima, M: On positive solutions of boundary value problems on the half-line. J. Math. Anal. Appl. 259, 127-136 (2001) 
10.1186/1687-2770-2013-27

Cite this article as: Sheng et al.: Existence and multiplicity of positive bound states for Schrödinger equations. Boundary Value Problems 2013, 2013:271

Submit your manuscript to a SpringerOpen ${ }^{\circ}$ journal and benefit from:

- Convenient online submission

- Rigorous peer review

- Immediate publication on acceptance

- Open access: articles freely available online

- High visibility within the field

- Retaining the copyright to your article

Submit your next manuscript at $\gg$ springeropen.com 